\title{
Deficits in Facial Emotion Recognition in Schizophrenia: A Replication Study with Korean Subjects
}

\author{
Seung Jae Lee ${ }^{1}$, Hae-Kook Lee ${ }^{2}$, Yong-Sil Kweon², Chung Tai Lee ${ }^{2}$ and Kyoung-Uk Lee ${ }^{2} \bowtie$ \\ ${ }^{1}$ Department of Psychiatry, Kyungpook National University School of Medicine, Daegu, Korea \\ 2Department of Psychiatry, Uijeongbu St. Mary's Hospital, The Catholic University of Korea College of Medicine, Uijeongbu, Korea
}

Objective We investigated the deficit in the recognition of facial emotions in a sample of medicated, stable Korean patients with schizophrenia using Korean facial emotion pictures and examined whether the possible impairments would corroborate previous findings.

Methods Fifty-five patients with schizophrenia and 62 healthy control subjects completed the Facial Affect Identification Test with a new set of 44 colored photographs of Korean faces including the six universal emotions as well as neutral faces.

Results Korean patients with schizophrenia showed impairments in the recognition of sad, fearful, and angry faces $[\mathrm{F}(1,114)=6.26$, $\mathrm{p}=0.014 ; \mathrm{F}(1,114)=6.18, \mathrm{p}=0.014 ; \mathrm{F}(1,114)=9.28, \mathrm{p}=0.003$, respectively], but their accuracy was no different from that of controls in the recognition of happy emotions. Higher total and three subscale scores of the Positive and Negative Syndrome Scale (PANSS) correlated with worse performance on both angry and neutral faces. Correct responses on happy stimuli were negatively correlated with negative symptom scores of the PANSS. Patients with schizophrenia also exhibited different patterns of misidentification relative to normal controls.

Conclusion These findings were consistent with previous studies carried out with different ethnic groups, suggesting cross-cultural similarities in facial recognition impairment in schizophrenia.

Psychiatry Investig 2010;7:291-297

Key Words Facial, Emotion, Recognition, Korean, Schizophrenia.

\section{INTRODUCTION}

Impaired recognition of facial affect in schizophrenia has been documented extensively. ${ }^{1,2}$ Specifically, rather than a general deficit that encompasses all emotions, schizophrenia may be associated with a more specific deficit in the processing of a subset of negative emotions. ${ }^{3}$ For example, individuals with schizophrenia showed normal performance in the recognition of happy faces, but they were significantly impaired in the recognition of anger, sadness, and fear. ${ }^{4-6}$ Relationships between this deficit and clinical variables have been reported, including schizophrenia subtypes ${ }^{7}$ and symptoms, ${ }^{7-10}$ and cognitive $e^{8,11}$ and social functioning. ${ }^{12}$

Received: December 22, 2009 Revised: April 1, 2010

Accepted: April 6, 2010 Available online: November 23, 2010

$\square$ Correspondence: Kyoung-Uk Lee, MD, PhD

Department of Psychiatry, Uijeongbu St. Mary's Hosptial, The Catholic University of Korea College of Medicine, 65-1 Geumo-dong, Uijeongbu 480-130, Korea

Tel: +82-31-820-3609, Fax: $+82-31-847-3630$

E-mail: mindcure@catholic.ac.kr

(a) This is an Open Access article distributed under the terms of the Creative Commons Attribution Non-Commercial License (http://creativecommons.org/licenses/by$\mathrm{nc} / 3.0$ ) which permits unrestricted non-commercial use, distribution, and reproduction in any medium, provided the original work is properly cited.
Although schizophrenia appears quite to be similar across a range of cultures, cross-cultural variability has been noted. ${ }^{13}$ There have been a few cross-cultural studies of facial emotion recognition comparing schizophrenia patients and normal controls. ${ }^{3,14-16}$ Brekke et al. ${ }^{14}$ reported that Caucasians with schizophrenia performed better on a facial emotion perception task than did African-American and Latino patients. Similarly, Habel et al. ${ }^{15}$ used stimuli consisting of Caucasian faces and found that face discrimination performance was impaired across patient groups (Americans, Germans, and Indians), but was most impaired among those of Indian origin. Matsumoto ${ }^{16}$ also showed that healthy Japanese subjects performed worse in the identification of Caucasian facial emotions than healthy American subjects did in the identification of Japanese facial emotions. More recently, Pinkham et al., ${ }^{17}$ using both Caucasian and African-American faces as stimuli, demonstrated the other-race effect, namely that participants with schizophrenia were more likely to recognize same-race faces than other-race faces. However, the majority of the studies have focused on Caucasian samples using only Caucasian faces as stimuli, and culture or ethnic-specific data on facial emotion recognition, especially in schizophrenia, are still scarce. 
Although several studies in Korea have also investigated the ability to recognize facial emotion in schizophrenia, using either non-Korean or less well-validated facial stimuli, these researchers did not fully demonstrate the same-race effect. ${ }^{18,19}$ Moreover, Biehl et al. ${ }^{20}$ reported that different Asian ethnic groups showed differences in the recognition of facial expression, suggesting that grouping the countries according to a Western/Non-Western dichotomy was not justified.

The most widely used facial stimuli for evaluating emotion recognition performance were developed by Ekman and Friesen. ${ }^{21}$ Because their facial pictures did not include Asian faces, causing some restriction in ethnicity, Matsumoto and Ek$\operatorname{man}^{22}$ developed a set of facial emotion stimuli consisting of an equal number of Caucasian and Japanese faces. In Korea, our group has developed and standardized a new set of 44 colored photographs of Korean faces, called the Facial Affect Identification Test (FAIT), which includes the six universal emotions as well as neutral faces. ${ }^{23}$ The mean concurrent validity for each emotion ranged from $76 \%$ to $99 \%$, except for the emotions of disgust and panic.

In the present study, we investigated deficits in the recognition of facial emotions in a sample of medicated, stable Korean patients with schizophrenia using Korean facial emotion pictures and examined whether the possible impairments would corroborate previous findings. Specifically, neutral faces were included to see if patients with schizophrenia misidentified neutral cues as unpleasant or threatening relative to normal controls.

\section{METHODS}

\section{Subjects}

Fifty-five patients with schizophrenia ( 24 men, 31 women) were recruited from Uijeongbu St. Mary's Hospital and Kyungpook National University Hospital, and 62 healthy control subjects (29 men, 33 women) were recruited from community settings. After receiving a complete description of the study, written informed consent was obtained from all participants. This study was approved by the Institutional Review Board of Kyungpook National University Hospital.

Patients were clinically stable in- or out-patients without prominent positive symptoms at the time of the study. The diagnosis of schizophrenia was established by experienced psychiatrists according to the DSM-IV. Detailed chart reviews were conducted to rule out patients with mental retardation, with a history of substance use disorder, and with any neurologic and medical disorders known to influencing cognitive functioning.

Regarding antipsychotic medications, 49 patients were taking stable regimens of atypical antipsychotics (amisulpride, aripiprazole, clozapine, olanzapine, quetiapine, or risperidone), and six were taking stable regimens of typical antipsychotics (haloperidol or fluphenazine) at therapeutic dosages. Healthy participants were recruited from staff members in the two hospitals mentioned above. Subjects were screened for psychotic, mood, and substance use disorders as well as for a history of head injury with significant loss of consciousness (greater than $5 \mathrm{~min}$ ) or neurological disorder.

Demographic data for both groups and symptom characteristics of the schizophrenic patients are shown in Table 1. The two groups did not differ significantly in terms of sex and age, but years of education were significantly lower for patients with schizophrenia than for normal controls. Assessment of the Positive and Negative Syndrome Scale (PANSS) at the time of testing revealed low levels of positive and negative psychotic symptoms and general psychopathology in patients as shown in (Table 1).

\section{Facial Affect Identification Test}

FAIT is a computerized test that uses ChaeLee Korean facial expressions of emotions including happiness, sadness, fear, anger, disgust, surprise, and neutral as stimuli. Methods of its development and validation were reported elsewhere. ${ }^{23}$ Briefly, three professional actors and three actresses participated in the development. They were asked to pose each of the following facial expressions: happiness, sadness, fear, anger, disgust, and surprise. To validate the identifiability of the intended emotion, hundreds of photographs were presented to five healthy raters, and 44 of these were finally chosen on the basis of the consistency of judgments. Following that, the validity of photographs was tested by 100 individuals.

In the current study, subjects were given an explanation regarding each emotion and had two practice sessions before the FAIT began. Then, subjects were asked to choose one of six emotions or a neutral expression while viewing the facial imag-

Table 1. Demographic and clinical data for schizophrenia and control groups

\begin{tabular}{lccc}
\hline & $\begin{array}{c}\text { Schizophrenia } \\
(\mathrm{N}=55)\end{array}$ & $\begin{array}{c}\text { Normal controls } \\
(\mathrm{N}=62)\end{array}$ & Statistics \\
\hline Male/Female (N) & $24 / 31$ & $29 / 33$ & $\chi^{2}=0.16$ \\
Age (year) & $32.1(8.9)$ & $32.7(9.8)$ & $\mathrm{t}=-0.22$ \\
Education (year) & $12.7(2.4)$ & $16.7(3.0)$ & $\mathrm{t}=-8.04^{*}$ \\
PANSS & & & \\
$\quad$ Positive & $12.8(4.9)$ & - & \\
$\quad$ Negative & $12.0(3.6)$ & - & \\
$\quad$ General & $28.2(7.0)$ & - & \\
$\quad$ Total score & $53.0(13.9)$ & - & \\
Years since first & $8.2(5.9)$ & - & \\
hospitalization & & & \\
\hline
\end{tabular}

Standard deviations appear in parentheses. ${ }^{*} \mathrm{p}<0.001$. PANSS: Positive and Negative Syndrome Scale 
es. A total of 44 facial images displaying happy, sad, fearful, angry, surprised, disgusted, or neutral expressions were then presented: seven faces expressed surprise and disgust, and six faces expressed the other emotions and neutral expressions. Hence, the maximum score was either six or seven according to the number of facial images presented for each emotion. The order of stimuli was randomized. The choices were displayed on the screen along with the stimuli, and subjects responded by pressing the touch screen.

\section{Data analysis}

The chi-square test (gender) and the t-test (age, education) were used to compare demographic characteristics between the schizophrenia and normal control groups. For the analysis of between-group differences on the FAIT, analysis of covariance (ANCOVA) was used for overall correct responses, with education as a covariate, and multivariate analysis of covariance (MANCOVA) was used for each emotion, with education as a covariate. Analysis of the total mean response time as well as the mean response time to an individual emotion was carried out in the same manner. Pearson's correlations were performed to detect the relationship between psychiatric symptoms and performance on the FAIT in the schizophrenia group. Fisher's exact test was also used to compare error patterns between the two groups.

Data were analyzed using PASW Statistics, version 18 (SPSS, Inc.; Chicago, IL, USA). The significance level was established at 0.05 .

\section{RESULTS}

\section{Emotion recognition}

The ANCOVA, with education as a covariate, revealed that patients with schizophrenia $(29.2 \pm 4.9)$ correctly identified the overall emotion of facial stimuli less often than did controls $(32.5 \pm 3.7)[\mathrm{F}(1,114)=6.33, \mathrm{p}=0.013]$. The MANCOVA revealed that schizophrenic patients preformed worse than controls in the recognition of sadness, fear, and anger $[\mathrm{F}(1,114)=6.26$, $\mathrm{p}=0.014 ; \mathrm{F}(1,114)=6.18, \mathrm{p}=0.014 ; \mathrm{F}(1,114)=9.28, \mathrm{p}=0.003$, respectively](Figure 1).

\section{Response time}

Schizophrenic patients showed significantly longer overall mean response times to facial stimuli than did controls (5.50 \pm $2.5 \mathrm{sec}$ for schizophrenic patients; $3.10 \pm 1.4 \mathrm{sec}$ for controls) [F $(1,114)=17.38, \mathrm{p}<0.001]$. Relative to healthy participants, schizophrenic patients showed a delayed response to all emotional and neutral faces, except fearful faces (Table 2). Note that the response time of participants as a whole varied across emotions from $1.01 \pm 0.6$ for anger to $6.01 \pm 3.3$ for surprise.

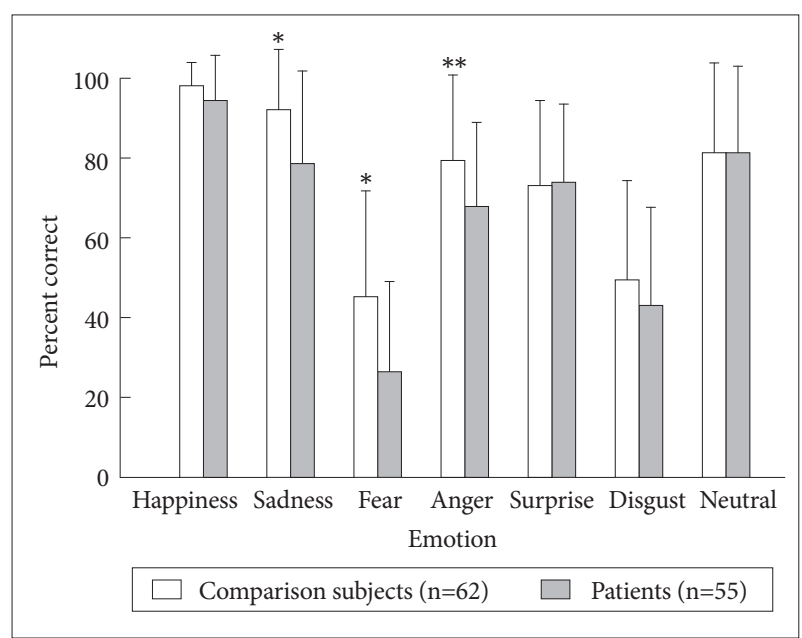

Figure 1. Recognition of facial emotions by patient with schizophrenia and normal controls. ${ }^{*} p<0.05,{ }^{* *} p<0.01$.

Table 2. Response time (second)

\begin{tabular}{lcclrcc}
\hline & \multicolumn{2}{c}{ Mean (SD) } & & \multicolumn{2}{c}{ Statistics } \\
\cline { 2 - 3 } \cline { 6 - 7 } & Schizophrenia & $\begin{array}{l}\text { Normal } \\
\text { controls }\end{array}$ & & $\mathrm{F}$ & $\mathrm{p}$ \\
\hline Happiness & $4.4(2.0)$ & $2.5(0.9)$ & & 26.4 & $<0.001$ \\
Sadness & $5.9(3.3)$ & $2.9(3.2)$ & & 12.7 & 0.001 \\
Fear & $5.9(3.9)$ & $4.1(2.8)$ & & 2.2 & 0.14 \\
Anger & $1.3(0.6)$ & $0.7(0.4)$ & & 20.6 & $<0.001$ \\
Surprise & $7.5(3.5)$ & & $4.7(2.6)$ & & 6.5 & 0.012 \\
Disgust & $6.8(3.0)$ & $3.7(2.6)$ & & 13.5 & $<0.001$ \\
Neutral & $6.8(4.8)$ & $3.5(1.9)$ & & 10.3 & 0.002 \\
Total & $5.5(2.5)$ & $3.1(1.4)$ & & 17.4 & $<0.001$ \\
\hline
\end{tabular}

\section{Relationship between the emotion recognition performance and clinical variables}

The correlations between correct response on the FAIT and symptom severity were significant for the total score as well as the three subscale scores of the PANSS. Within emotions, higher total and three subscale scores of the PANSS correlated with worse performance on both angry and neutral faces. The correct responses on the happy stimuli were negatively correlated with negative symptoms on the PANSS. However, only the correlation between angry faces and the PANSS was significant when the Bonferroni correction for multiple comparisons was applied [ $\alpha$-adjusted p level=0.0018 (0.05/28)](Table 3).

No significant correlations between the mean overall response time on the FAIT and the total as well as three subscale scores of the PANSS were found. However, the correlation between the overall response time and negative symptoms reached significance $(r=0.262, p=0.053)$. Within emotions, higher negative subscale scores on the PANSS were correlated with longer response times for sad, angry, and neutral faces. However, the correlations were not significant when the Bonferro- 
Table 3. Relationships between psychiatric symptoms and correct response of facial recognition test within schizophrenia group $(\mathrm{N}=55)$

\begin{tabular}{lllll}
\hline & \multicolumn{4}{c}{ Positive and Negative Syndrome Scale } \\
\cline { 2 - 5 } & Positive & Negative & General & Total \\
\hline Happiness & -0.241 & $-0.284^{*}$ & -0.199 & -0.259 \\
Sadness & -0.079 & -0.260 & -0.118 & -0.194 \\
Fear & -0.166 & -0.198 & -0.117 & -0.160 \\
Anger & $-0.472^{* *}$ & $-0.380^{*}$ & $-0.457^{* *}$ & $-0.475^{* *}$ \\
Surprise & -0.144 & -0.174 & -0.047 & -0.140 \\
Disgust & 0.120 & 0.093 & 0.140 & 0.131 \\
Neutral & $-0.272^{*}$ & $-0.370^{* *}$ & $-0.368^{* *}$ & $-0.388^{* *}$ \\
Total & $-0.293^{*}$ & $-0.378^{* *}$ & $-0.273^{*}$ & $-0.353^{* *}$ \\
\hline
\end{tabular}

${ }^{*} \mathrm{p}<0.05,{ }^{* *} \mathrm{p}<0.01$

Table 4. Relationships between psychiatric symptoms and response time of facial recognition test within schizophrenia group $(\mathrm{N}=55)$

\begin{tabular}{lcccc}
\hline & \multicolumn{3}{c}{ Positive and Negative Syndrome Scale } \\
\cline { 2 - 5 } & Positive & Negative & General & Total \\
\hline Happiness & 0.166 & 0.211 & 0.135 & 0.197 \\
Sadness & 0.093 & $0.365^{* *}$ & 0.160 & 0.221 \\
Fear & 0.010 & 0.090 & -0.035 & 0.038 \\
Anger & 0.111 & $0.305^{*}$ & 0.258 & 0.246 \\
Surprise & 0.075 & 0.088 & 0.073 & 0.100 \\
Disgust & 0.110 & 0.212 & 0.199 & 0.220 \\
Neutral & 0.108 & $0.293^{*}$ & 0.129 & 0.192 \\
Total & 0.110 & 0.262 & 0.134 & 0.196 \\
\hline
\end{tabular}

${ }^{*} \mathrm{p}<0.05,{ }^{* *} \mathrm{p}<0.01$

ni correction for multiple comparisons was applied [ $\alpha$-adjusted p level $=0.0018(0.05 / 28)]$ (Table 4$)$.

Duration of illness was not correlated with total correct response $(\mathrm{r}=-0.038, \mathrm{p}=0.545)$ or with response time $(\mathrm{r}=0.136$, $\mathrm{p}=0.323)$.

\section{Pattern of error rates}

We found that schizophrenic patients and healthy subjects differed in the distribution of errors for angry $(\mathrm{p}=0.014)$, surprised $(\mathrm{p}<0.001)$, disgusted $(\mathrm{p}=0.012)$, and neutral expressions $(\mathrm{p}<0.001$; all by Fisher's exact test). In general, whereas error responses in healthy participants were related to one prominent emotion, those in schizophrenic patients were relatively divergent. Schizophrenic patients were less likely to attribute disgust to angry expressions ( $21 \%$ for schizophrenic patients compared with $67 \%$ for healthy subjects), fearful to surprised expressions ( $46 \%$ versus $74 \%$ respectively) and sadness to disgusted expressions (66\% versus $79 \%$ respectively) compared with normal controls. Interestingly, schizophrenic patients were more likely to attribute happiness to neutral expressions (28\% versus $3 \%$ respectively) than were controls. In both groups, sad faces were most commonly misrecognized as disgusted, followed by misrecognizing angry expressions. Fearful faces were most commonly misrecognized as surprised, followed by misrecognizing angry expressions. Angry faces were most commonly misrecognized as disgusted, followed by misrecognizing fearful expressions. Surprised faces were most commonly misrecognized as fearful, followed by misrecognizing angry expressions. Disgusted faces were most commonly misrecognized as sad, followed by misrecognizing fearful expressions. Neutral faces were most commonly misrecognized as sad expressions ( $\mathrm{Ta}$ ble 5).

\section{DISCUSSION}

Impaired recognition of facial affect in schizophrenia has been investigated extensively. However, few studies have used color photographs of Korean faces with moderate sample size to investigate these impairments among Korean patients with schizophrenia. In this study, Korean patients with schizophrenia showed impairments in the recognition of sad, fearful, and angry faces, but they were as accurate as controls in the recognition of happy emotions. Patients with schizophrenia also exhibited different patterns of misidentification relative to normal controls. These findings were consistent with previous studies carried out with different ethnic groups, suggesting crosscultural similarities in the impairment of facial recognition in schizophrenia.

Schizophrenic patients in this study were found to be less accurate in the recognition of sad, fearful, and angry facial expressions. Note that these results were obtained regardless of the inherent difficulties of the given emotion. For example, the correct response rate for sad expressions was $93 \%$, whereas that for fearful expressions was just below $50 \%$ even in normal controls. These rates corroborated our initial validation study ${ }^{23}$ and Japanese data. ${ }^{16,22-24}$ On the other hand, schizophrenic patients did not differ from controls in recognizing happy (positive emotion) as well as surprise and disgusted expressions (negative emotions). Although it has been debated, ${ }^{25,26}$ our findings support the emotion-specific-impairment view in schizophrenia, which postulates a greater deficit for negative emotions and, furthermore, more specific deficits in the processing of a subset of negative emotions. ${ }^{4-6}$

As to our result showing a trend toward an association between response time and negative symptoms, delayed response times in schizophrenia can be accounted for by many confounding factors such as negative symptoms, persistent illness, medication induced movement disorders, and impaired general cognitive functioning. ${ }^{27}$ However, these findings might also reflect these patients' difficulties in recognizing facial emotions. 


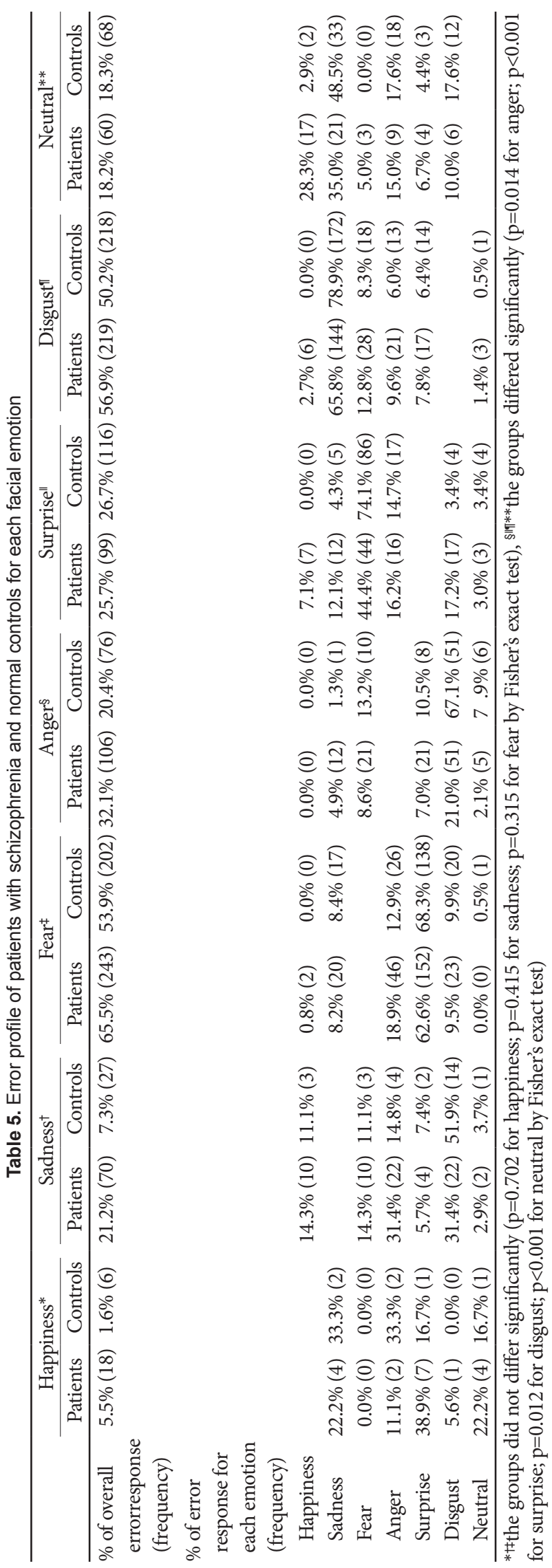

In particular, the mean response time for the recognition of the fearful faces was not significantly different between the two groups not because schizophrenic patients responded as rapidly as normal controls, but because normal controls exhibited delayed responses as did schizophrenia patients due to higher difficulty of recognizing the fearful faces.

With regard to the correlations between recognition and symptoms, worse performance on the facial emotion recognition test was associated with more severe symptoms, exhibiting a stronger relationship with negative symptom scores. A large body of previous literature, mostly using the Scale for the Assessment of Negative Symptoms, has indicated that negative symptoms affect the performance of facial emotion recognition in schizophrenic patients. ${ }^{3,6,8,9}$ Some studies reported a correlation between emotion recognition and positive symptoms. ${ }^{7.8}$ These mixed findings may partly depend on the clinical status of patients with schizophrenia. Although one study observed that patients with acute schizophrenia were more impaired in emotion recognition compared with normal subjects and with patients with schizophrenia in remission, most studies have been conducted with chronic and/or clinically stable patients. ${ }^{28}$ In the present study, emotional faces (i.e., happy, angry) as well as neutral faces were correlated with symptoms, which has implications for understanding symptoms in schizophrenia. Specifically, patients with schizophrenia frequently misidentify other's facial emotion in acute stage of their illness. ${ }^{9,29}$ Interestingly, difficulties in identifying happy emotions were related to the severity of negative symptoms. This evidence may support an inability to experience pleasure in activities, or anhedonia, in schizophrenia. ${ }^{30}$ Analysis of the patterns of error revealed consistent patterns in the misidentification of different emotional valences regardless of participant group. These observations were consistent with the study by Phillips et al., ${ }^{31}$ who reported surprise as the most common misidentification of fear, anger as the most common misidentification of disgust, and disgust as the most common misidentification of anger. Further findings were also confirmed by Johnston et al.s meticulous research reporting confusions between neutral and sad expressions. ${ }^{25}$ In general, the two groups differed in the patterns of error such that patients with schizophrenia showed more random distributions of errors than did normal controls. In this study, significant between-group differences in the pattern of erroneous misattributions were found not only for neutral faces, but also for expressions of anger, surprise, and disgust. These findings suggests that misattribution of facial emotion may not be limited to neutral expressions.

In this study, schizophrenic patients misidentified neutral cues as emotional, showing a negative as well as positive bias. Kohler et al. ${ }^{9}$ reported that patients with schizophrenia showed negative bias to neutral faces relative to controls, whereas Lep- 
pänen et al. ${ }^{3}$ found that patients exhibited more false happy (positive) as well as angry (negative) responses to neutral faces than did controls. A possible explanation for the happy (positive) response bias among schizophrenic patients may lie in the characteristics of study participants, who suffered chronically and were prone to show irrelevant responses. However, because of the lack of previous data on the analysis of the patterns of error rates and the use of a small and varied number of facial stimuli for each emotion in this study, these findings should be interpreted cautiously.

This study has some limitations. Impaired emotion recognition in this study was limited to a group of patients with schizophrenia who were clinically stable yet chronic. The effect of medication was not controlled in this study. Previous findings regarding this issue have been inconsistent. ${ }^{7}$ Although we controlled for the level of education, cognitive functions were not well controlled using more discrete variables. One similar study indicated that the facial emotion recognition was related not to general intelligence such as intelligence quotient, but to specific cognitive functions such as perseverative error. ${ }^{32}$ Our emotion-recognition task has some shortcomings, including a relatively small number of images for each emotion and the low correction rate for disgust and surprise ( $47 \%$ and $43 \%$ respectively). Also, the mean response time for each emotion varied regardless of participant group, which raised the issue of whether the expressional quantity of each condition was similar. Thus, cautious interpretation of the response time as absolute values is warranted.

In conclusion, using Korean emotional faces, we found that Korean patients with schizophrenia showed impairments in emotion recognition, specifically for sad, fearful, and angry faces. They also exhibited differences in the pattern of error rates. Previous studies with other ethnic groups as well as this study suggest cross-cultural similarities in the deficit in facial recognition in schizophrenia.

\section{REFERENCES}

1. Mandal MK, Pandey R, Prasad AB. Facial expressions of emotions and schizophrenia: a review. Schizophr Bull 1998;24:399-412.

2. Kohler CG, Martin EA. Emotional processing in schizophrenia. Cogn Neuropsychiatry 2006;11:250-271.

3. Leppänen JM, Niehaus DJ, Koen L, Du Toit E, Schoeman R, Emsley R. Emotional face processing deficit in schizophrenia: A replication study in a South African Xhosa population. Schizophr Res 2006;84:323-330.

4. Bediou B, Franck N, Saoud M, Baudouin JY, Tiberghien G, Daléry J, et al. Effects of emotion and identity on facial affect processing in schizophrenia. Psychiatry Res 2005;133:149-157.

5. Bell M, Bryson G, Lysaker P. Positive and negative affect recognition in schizophrenia: a comparison with substance abuse and normal control subjects. Psychiatry Res 1997;73:73-82.

6. Silver H, Shlomo N, Turner T, Gur RC. Perception of happy and sad facial expressions in chronic schizophrenia: evidence for two evaluative systems. Schizophr Res 2002;55:171-177.
7. Weniger G, Lange C, Rüther E, Irle E. Differential impairments of facial affect recognition in schizophrenia subtypes and major depression. Psychiatry Res 2004;128:135-146.

8. Kohler CG, Bilker W, Hagendoorn M, Gur RE, Gur RC. Emotion recognition deficit in schizophrenia: association with symptomatology and cognition. Biol Psychiatry 2000;48:127-136.

9. Kohler CG, Turner TH, Bilker WB, Brensinger CM, Siegel SJ, Kanes SJ, et al. Facial emotion recognition in schizophrenia: intensity effects and error pattern. Am J Psychiatry 2003;160:1768-1774.

10. Schneider F, Gur RC, Gur RE, Shtasel DL. Emotional processing in schizophrenia: neurobehavioral probes in relation to psychopathology. Schizophr Res 1995;17:67-75.

11. Sachs G, Steger-Wuchse D, Kryspin-Exner I, Gur RC, Katschnig H. Facial recognition deficits and cognition in schizophrenia. Schizophr Res 2004; 68:27-35.

12. Hall J, Harris JM, Sprengelmeyer R, Sprengelmeyer A, Young AW, Santos IM, et al. Social cognition and face processing in schizophrenia. $\mathrm{Br}$ J Psychiatry 2004;185:169-170.

13. Bae SW, Brekke JS. Characteristics of Korean-Americans with schizophrenia: a cross-ethnic comparison with African-Americans, Latinos, and Euro-Americans. Schizophr Bull 2002;28:703-717.

14. Brekke JS, Nakagami E, Kee KS, Green MF. Cross-ethnic differences in perception of emotion in schizophrenia. Schizophr Res 2005;77:289298.

15. Habel U, Gur RC, Mandal MK, Salloum JB, Gur RE, Schneider F. Emotional processing in schizophrenia across cultures: standardized measures of discrimination and experience. Schizophr Res 2000;42:57-66.

16. Matsumoto D. American-Japanese cultural differences in the recognition of universal facial expressions. J Cross Cult Psychol 1992;23:72-84.

17. Pinkham AE, Sasson NJ, Calkins ME, Richard J, Hughett P, Gur RE, et al. The other-race effect in face processing among African American and Caucasian individuals with schizophrenia. Am J Psychiatry 2008;165: 639-645.

18. Park SH, Yoon SA, Kim MS. Deficits of facial affect perception in patients with schizophrenia. Korean J Clin Psychol 2007;26:845-865.

19. Jin BS, Choi YS, Son MJ. Facial-emotion perception of the patients with schizophrenia. Korean J Clin Psychol 1998;17:197-209.

20. Biehl M, Matsumoto D, Ekman P, Hearn V, Heider K, Kudoh T, et al. Matsumoto and Ekman's Japanese and Caucasian Facial Expressions of Emotion (JACFEE): Reliability Data and Cross-National Differences. J Nonverbal Behav 1997;21:3-21.

21. Ekman P, Friesen WV. Pictures of facial affect. Palo Alto, CA: Consulting Psychologists' Press; 1976.

22. Matsumoto D, Ekman P. Japanese and Caucasian facial expressions of emotion (JACFEE) slides. San Francisco, CA: Intercultural and emotion research laboratory, department of psychology, San Francisco State University; 1988.

23. Lee WH, Chae JH, Bahk WM, Lee KU. Development and its preliminary standardization of pictures of facial expression for affective neurosciences. J Korean Neuropsychiatr Assoc 2004;43:552-558.

24. Russell JA. Is there universal recognition of emotion from facial expression? A review of the cross-cultural studies. Psychol Bull 1994;115: 102-141.

25. Johnston PJ, Katsikitis M, Carr VJ. A generalised deficit can account for problems in facial emotion recognition in schizophrenia. Biol Psychol 2001;58:203-227.

26. Mandal MK, Jain A, Haque-Nizamie S, Weiss U, Schneider F. Generality and specificity of emotion-recognition deficit in schizophrenic patients with positive and negative symptoms. Psychiatry Res 1999;87: 39-46.

27. Ngan ET, Liddle PF. Reaction time, symptom profiles and course of illness in schizophrenia. Schizophr Res 2000;46:195-201.

28. Gessler S, Cutting J, Frith CD, Weinman J. Schizophrenic inability to judge facial emotion: a controlled study. Br J Clin Psychol 1989;28:1929. 
29. Phillips ML, David AS. Facial processing in schizophrenia and delusional misidentification: cognitive neuropsychiatric approaches. Schizophr Res 1995;17:109-114.

30. Trémeau F. A review of emotion deficits in schizophrenia. Dialogues Clin Neurosci 2006;8:59-70.

31. Phillips ML, Williams L, Senior C, Bullmore ET, Brammer MJ, An- drew $\mathrm{C}$, et al. A differential neural response to threatening and nonthreatening negative facial expressions in paranoid and non-paranoid schizophrenics. Psychiatry Res 1999;92:11-31.

32. Lee SJ, Lee HK, Kweon YS, Lee CT, Lee KU. The impact of executive function on emotion recognition and emotion experience in patients with schizophrenia. Psychiatry Investig 2009;6:156-162. 